\title{
A rapid method for determining chlorobenzenes in dam water systems
}

\author{
KG Moodley ${ }^{1 *}$, DK Chetty ${ }^{1}$, SR Ramphal ${ }^{1}$ and G Gericke ${ }^{2}$ \\ ${ }^{1}$ Department of Chemistry, Durban University of Technology, Private Bag 1334, Durban, 4000, South Africa \\ ${ }^{2}$ Eskom Laboratory, Lower Germiston Road, Rosherville, Gauteng, South Africa
}

\begin{abstract}
A method using direct immersion solid phase microextraction (DI-SPME) coupled to gas chromatography equipped with a flame ionisation detector (GC-FID) was developed for the analysis of 7 chlorinated benzenes in dam water. The main parameters affecting the DI-SPME process were optimised. The optimised method comprises the use of a $100 \mu \mathrm{m}$ polydimethylsiloxane (PDMS) fibre coating; $5 \mathrm{~m} \ell$ sample size; $700 \mathrm{r} / \mathrm{min}$ rate of agitation and an extraction time of $30 \mathrm{~min}$. The calibration curve was linear with correlation coefficients ranging from $0.9957-0.9995$ for a concentration range of 1-100 $\mathrm{ng} / \mathrm{m} \ell$. The limits of detection and quantification ranged from $0.020-0.265 \mathrm{ng} / \mathrm{m} \ell$ and $0.204-2.65 \mathrm{ng} / \mathrm{m} \ell$, respectively. Recoveries ranged from 83.6-107.2\% with relative standard deviation of less than $9.2 \%$, indicating that the method has good precision. The method is reliable and is free of matrix interferences. Water samples collected from Grootdraai Dam were analysed using the optimised conditions to assess the potential of the method for trace level screening and quantification of chlorobenzenes. The method proved to be efficient, as 1,3 dichlorobenzene, 1,4-dichlorobenzene and pentachlorobenzene were detected at concentrations of $0.429 \mathrm{ng} / \mathrm{m} \ell, 1.685 \mathrm{ng} / \mathrm{m} \ell$ and $1.433 \mathrm{ng} / \mathrm{m} \ell$, respectively.
\end{abstract}

Keywords: chlorobenzenes, Grootdraai Dam, water, dam, SPME, DI-SPME, GC-FID

\section{Introduction}

Among persistent organic pollutants (POPs), chlorobenzenes are some of the most frequently encountered compounds in aqueous systems. These compounds can enter the environment via natural and anthropogenic sources, and are ubiquitous due to their extensive use over the past several decades (Soonthorntantikul et al., 2009). Several chlorobenzene compounds, once in the environment, can be biologically accumulated, and are reputed to be carcinogens and extremely hazardous to health. Chlorobenzene compounds are listed as priority pollutants by the United States Environmental Protection Agency (USEPA) (He et al., 2000). Excessive exposure to these compounds affects the central nervous system, irritates skin and upper respiratory tract, hardens skin and leads to haematological disorders including anaemia (Kozani et al., 2007; Khajeh et al., 2006). In spite of these harmful effects, chlorobenzenes are still used widely as process solvents and raw materials in the manufacture of pesticides, chlorinated phenols, lubricants, disinfectants, pigments and dyes (Grosjean, 1991; Vidal et al., 2007). In the light of the above, it is imperative to continuously monitor the levels of chlorinated benzenes in all types of surface waters, using a low-cost but sensitive method.

Analysis of chlorobenzenes at trace and ultra-trace levels requires pre-concentration as well as sample matrix clean-up to remove any interfering components (Chisvert et al., 2009). Various pre-concentration techniques have been used for the isolation of chlorobenzenes from water. These include traditional liquid-liquid extraction (Ormad et al., 1996); solid phase extraction (Wang and Lee, 1998; Liu et al., 2004); single drop

\footnotetext{
* To whom all correspondence should be addressed

용 +2731373 5133; fax: +27 31373 5317;

e-mail: $\underline{\text { moodlykg@dut.ac.za }}$
}

Received 16 April 2012; accepted in revised form 21 December 2012. microextraction (Tor, 2006; Khajeh et al., 2006; Vidal et al., 2005); stir bar sorptive extraction (Montero et al., 2004) and solid phase micro-extraction (SPME) (He et al., 2000; Wang et al., 2003; Huang et al., 2007). These pre-concentration steps precede analysis using gas chromatography coupled to an appropriate detector for identification. Whereas mass spectroscopy (MS) and electron capture detectors (ECD) are excellent detectors for the analysis of these compounds, due to superior sensitivity, accuracy and resolution, they incur relatively high capital, operational and maintenance costs which results in decreased throughput. Although relatively low detection limits can be attained with MS and ECD as detectors, the actual limits set by environmental protection agencies are much higher and can be attained with less expensive systems. This report describes a system comprising a pre-concentration device, a standard chromatograph and a flame ionisation detector (FID). Pre-concentration was effected through direct immersion solid phase micro-extraction (DI-SPME).

Experimental parameters affecting the extraction efficiency of the selected chlorobenzenes, such as fibre type, sample size, rate of agitation, salting-out effect and extraction time, were optimised and applied to the Grootdraai Dam water samples for the quantification of chlorobenzenes.

\section{Experimental}

\section{Reagents and materials}

The chlorobenzenes selected for this study were: 1,2-dichlorobenzene (1,2-DCB); 1,3-dichlorobenzene (1,3-DCB);

1,4-dichlorobenzene (1,4-DCB); 1,2,4-trichlorobenzene (1,2,4TCB); 1,2,3,4-tetrachlorobenzene (1,2,3,4-TeCB); 1,2,4,5-tetrachlorobenzene (1,2,4,5-TeCB) and pentachlorobenzene (PCB). These standards were high purity $(>98 \%)$ and were purchased together with HPLC grade methanol (99.9\%) from Sigma Aldrich (Milwaukee, WI, USA). 'ACS reagent' grade sodium 


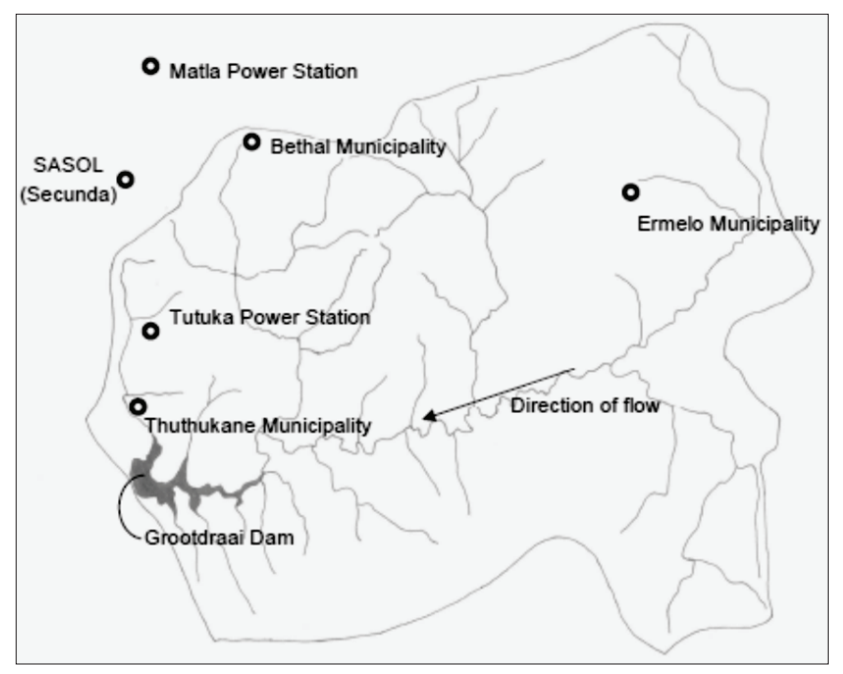

Figure 1

Grootdraai Dam catchment (Adapted from Strauss, 2006)

chloride (>99\%) was purchased from Merck Darmstadt, Germany. $100 \mu \mathrm{m}$ polydimethylsiloxane (PDMS) and $85 \mu \mathrm{m}$ polyacrylate (PA) SPME fibres were purchased from Supelco (Bellefonte, PA, USA). $1000 \mathrm{mg}$ stock solutions of individual standards were prepared. A $1000 / \ell \mathrm{mg} / \ell$ multi-compound solution was prepared, from which a $100 \mu \mathrm{g} / \ell$ working standard was prepared daily by a series of dilutions. All solutions were prepared in methanol and stored under refrigeration at $4^{\circ} \mathrm{C}$. Purified water $(18 \mathrm{M} \Omega \cdot \mathrm{cm}$ resistivity) for this study was taken from a Milli-Q water purification system (Millipore, Bedford, MA, USA).

\section{Sampling}

Grab samples were taken from the Grootdraai Dam in $1 \ell$ Schott bottles, which were subsequently stored under refrigeration at $4{ }^{\circ} \mathrm{C}$. The samples were taken at the dam wall and from a depth of approximately $0.5 \mathrm{~m}$. No samples were taken from the point of discharge. The Grootdraai water samples were filtered through a $0.45 \mu \mathrm{m}$ membrane filter, prior to analysis, to eliminate particulate matter. The Grootdraai Dam is an impoundment on the Vaal River which is situated northeast of Standerton in the province of Mpumalanga, South Africa (locality: 26 54'33.6”S $29^{\circ} 23^{\prime} 42.5^{\prime \prime}$ ) (Van Ginkel, 2001). The dam, built in 1981, has a gross storage capacity of 364 million $\mathrm{m}^{3}$, surface area of $39 \mathrm{~km}^{2}$ and an average depth of $27 \mathrm{~m}$. The Grootdraai Dam is upstream of the Vaal Dam, forming part of the Usuthu-Vaal Scheme (Strauss, 2006; Jeleni and Mare, 2007).

With the exception of a small local community, there is limited domestic use of water directly from the dam. The Grootdraai Dam catchment has a surface area of $7928 \mathrm{~km}^{2}$ and the major water users are industries and municipalities (Fig. 1) (Strauss, 2006; Van Ginkel, 2001).

\section{SPME procedure}

The optimisation of the main parameters influencing an SPME extraction was performed using Millipore water fortified with the seven chlorobenzene isomers. All SPME extractions were performed in $22 \mathrm{~m} \ell$ amber glass vials. For a typical experiment, an aliquot of $5 \mathrm{~m} \ell$ of Millipore water was fortified with a mass of $10 \mathrm{ng}$ of each analyte. In the case of the experiments for salting-out effect, $5 \mathrm{~m} \ell$ sodium chloride solutions of $5 \%$, $10 \%, 15 \%$ and $20 \%(\mathrm{~m} / \mathrm{v})$ instead of pure Millipore water were used. To enhance the transfer of analytes to the fibre, agitation was performed using a $2 \mathrm{~cm}$ Teflon stir bar, coupled to a magnetic stirrer. The vials were then sealed with screw-type plastic caps containing a PTFE/silicon septum. The SPME manual holder needle was used to penetrate the septum, and the fibre was then directly immersed into the sample solution for a pre-determined time. To enhance exposure of the fibre to the analytes, precaution was taken to ensure that the fibre was exposed to the outside flow of the vortex rather than directly within the vortex. After extraction, the fibre was retracted into the protective needle and removed from the sample vial. Lint free tissue paper was used to remove any water molecules present at the entrance of the protective needle. The protective needle was then used to penetrate the septum of the GC, and the fibre was then exposed to thermal conditions of the injector port for desorption. All samples were freshly prepared minutes before the extraction to reduce/eliminate any losses due to volatilisation or decomposition due to prolonged storage. SPME fibres were conditioned for 30 min every morning and blank fibre injections were performed to assess the presence of any contamination within the fibre.

\section{GC parameters}

Analysis was performed with a Shimadzu (Tokyo, Japan) GC-2014 GC-FID system equipped with a SBPX-5 capillary column ( $30 \mathrm{~m}$ x $0.25 \mathrm{~mm}$ internal diameter, $0.25 \mu \mathrm{m}$ film thickness). Helium was used as the carrier gas which was passed through an oxygen, moisture and hydrocarbon trap prior to entering the GC. All GC injections were done in the splitless mode and the injector port was equipped with a glass insert for splitless injection. The vent valve of the split outlet was closed for $30 \mathrm{~s}$ and then opened fully. The GC conditions were as follows: injector port and FID temperatures were $250^{\circ} \mathrm{C}$ and $300^{\circ} \mathrm{C}$, respectively; constant column flow rate was $1 \mathrm{m \ell} / \mathrm{min}$; oven temperature programme was $60^{\circ} \mathrm{C}$ held for 2 minutes, ramped at $10^{\circ} \mathrm{C} / \mathrm{min}$ to $70^{\circ} \mathrm{C}$ and held for $4 \mathrm{~min}$, ramped at $5^{\circ} \mathrm{C} / \mathrm{min}$ to $85^{\circ} \mathrm{C}$, ramped at $15^{\circ} \mathrm{C} / \mathrm{min}$ to $205^{\circ} \mathrm{C}$, ramped at $25^{\circ} \mathrm{C} / \mathrm{min} 280^{\circ} \mathrm{C}$ and held for $5 \mathrm{~min}$. The total analysis time was $26 \mathrm{~min}$. All chromatographic data were processed using the GC solution post-run software (Shimadzu, version 2.30).

\section{Results and discussion}

\section{Optimisation of parameters in the DI-SPME of chlorobenzenes}

Several analytical parameters can be optimised to enhance the extraction efficiency of chlorobenzenes from water using the DI-SPME method. These parameters included SPME fibre coating, sample size, rate of agitation, salting-out effect and extraction time.

\section{SPME fibre coating}

Fibres coated with polyacrylate (PA) and polydimethlylsiloxane (PDMS) were tested for extraction efficiency. The results, depicted in Fig. 1, show that the PDMS-coated fibre gives better extraction for all seven analytes tested. This is also consistent with the fact that PDMS is more non-polar than PA and that the analytes have relatively low polarity. The PDMS fibre was used for the rest of the study. 


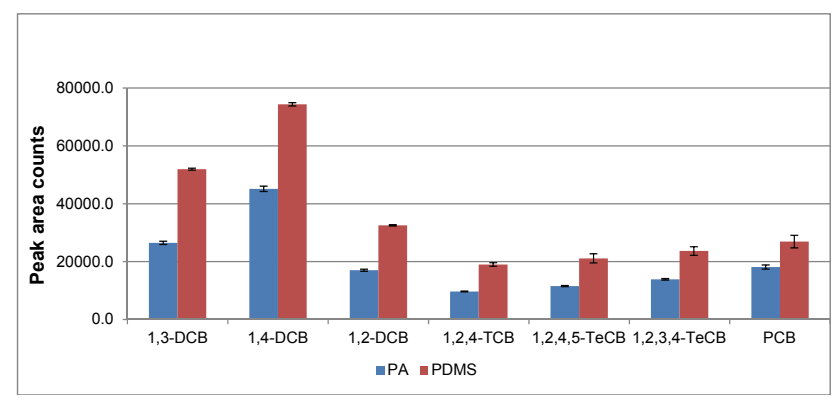

Figure 2

Extraction efficiencies of the PA and PDMS coating; error bars indicate standard error of experiment $(n=3)$

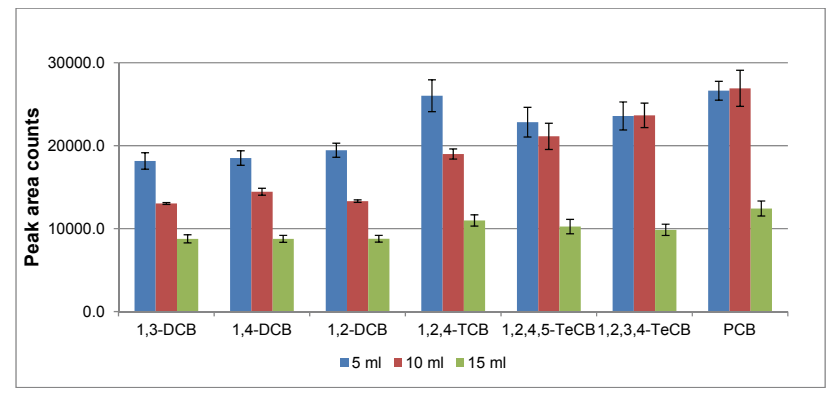

Figure 3

Influence of sample size on extraction efficiency, error bars indicates standard error of experiment $(n=3)$

\section{Sample size}

In DI-SPME studies performed on water matrices, such as those of Filho et al. (2010), Lopez-Darias et al. (2010a), LopezDarias et al. (2010b), Meng and Anderson, (2010), Li et al. (2009), Luan et al. (2007) and Junior and Re-Poppi (2007), a constant sample size was used while optimising all other parameters. Since this study aimed to take advantage of any enhancement in extraction efficiency, 3 sample volumes $(5 \mathrm{~m} \ell$, $10 \mathrm{~m} \ell$ and $15 \mathrm{~m} \ell$ ) were investigated.

Figure 3 shows that superior extraction efficiency was obtained using a sample volume of $5 \mathrm{~m} \ell$. Further increases in sample volume resulted in decreased extraction efficiency. The enhanced extraction efficiency at lower sample volume can be attributed to a larger phase ratio between volume of the fibre coating and sample. In light of the above, a sample size of $5 \mathrm{~m} \ell$ was used for the rest of the study.

\section{Effect of rate of agitation}

In view of the observation (Pawliszyn, 1997) that equilibration times are dependent on the rate at which the solution is stirred, 4 stir speeds were tested while other parameters were kept constant. The results are shown in Fig. 4. It is evident that a stir speed of $700 \mathrm{r} / \mathrm{min}$ resulted in the highest extraction efficiency; while further increase to $1100 \mathrm{r} / \mathrm{min}$ resulted in a decrease in extraction efficiency. Kin (2008) experienced a similar phenomenon, which is due to the vibration of the stir bar and the irregular agitation of the sample at high stir speeds. Furthermore, a stir rate of $1100 \mathrm{r} / \mathrm{min}$ resulted in splashing of sample onto the walls of the glass vial. This could have resulted in the loss of analytes and decreased extraction efficiency. In addition, higher random errors were obtained at higher stir rates, indicating that experiments at this stir speed were a lot more difficult to reproduce, thereby decreasing method precision.

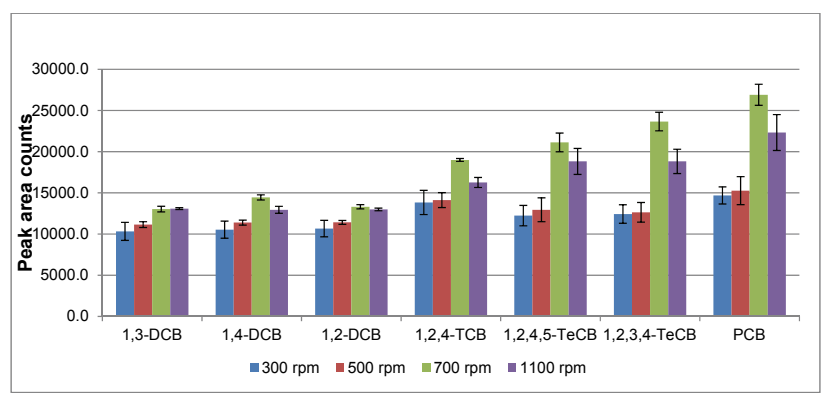

Figure 4

Influence of rate of agitation on extraction efficiency; error bars indicate standard error of experiment $(n=3)$

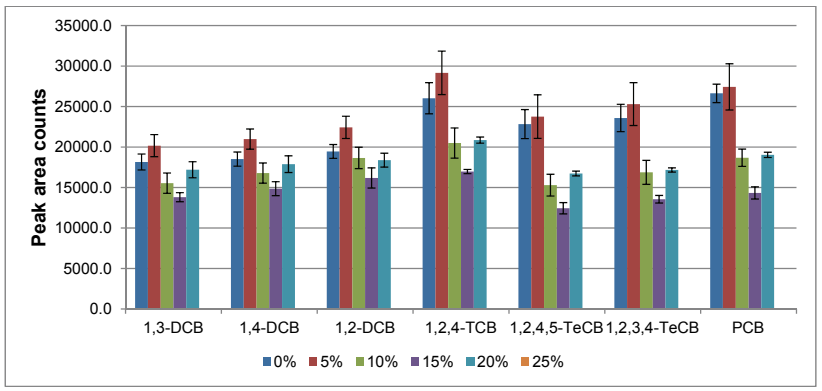

Figure 5

Impact of salting-out effect on extraction efficiency; error bars indicate standard error of experiment $(n=3)$

From Fig. 4, it is apparent that the rate of agitation had a minimum effect on the lower chlorinated benzenes (1,3-DCB, 1,2-DCB and 1,4-DCB, 1,2,4-TCB), while higher stir rates promoted an enhanced extraction efficiency for the higher chlorinated benzenes (1,2,4,5- and 1,2,3,4-TeCB and PCB). This suggests that the rate of mass transfer from the bulk of the sample to the fibre is sufficient for low molecular weight chlorobenzenes at low rates of agitation. The converse is true of high molecular weight chlorobenzenes; therefore a stir speed of $700 \mathrm{r} / \mathrm{min}$ was deemed optimum and was used for the rest of the study.

\section{Salting-out effect}

Salt is added, in SPME, with the aim of effecting a modification to the sample matrix. An increase in ionic strength reduces the solubility of some analytes. A concomitant increase in extraction efficiency is expected on the basis that more of the analyte is available for adsorption onto the fibre (Kin, 2008). As such, sodium chloride was added to aqueous samples to attain concentrations $(\mathrm{m} / \mathrm{v})$ ranging from $0-20 \%$.

Figure 5 indicates that $5 \%(\mathrm{~m} / \mathrm{v})$ salt concentration had a slight improvement on the extraction efficiency while a further increase in salt concentration resulted in a drastic decrease in extraction efficiency. Ionic strength has a greater effect on analytes which have high water solubility (Santos et al., 1996). Therefore, it is evident that the compounds with higher water solubility (1,2-DCB, 1,3-DCB, 1,4-DCB and 1,2,4-TCB) were extracted to a greater extent $(9-13 \%)$. By contrast, ionic strength had a minimal influence on compounds with lower water solubility $(1,2,3,4-\mathrm{TeCB} ; 1,2,4,5-\mathrm{TeCB}$ and $\mathrm{PCB})$ resulting in a $2-6 \%$ enhancement in extraction efficiency. These observations are attributed to the effect of hydration spheres around the salt molecules. These hydration spheres reduce the 


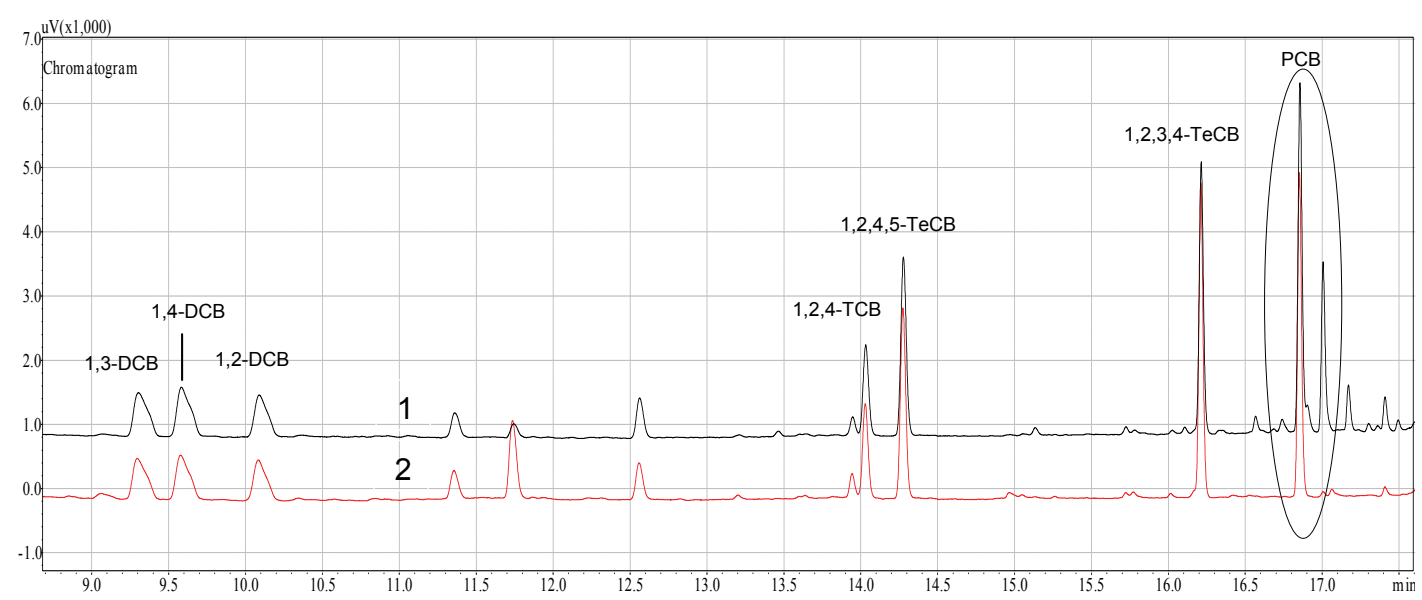

Figure 6 (above)

Interfering compound due to the addition of salt; (1) extraction with the addition of $5 \%(\mathrm{~m} / \mathrm{v})$ salt; (2) extraction with $0 \%(\mathrm{~m} / \mathrm{v})$ salt

Figure 7(right) The effect of extraction time on extraction efficiency; error bars indicate standard error of experiment $(n=3)$

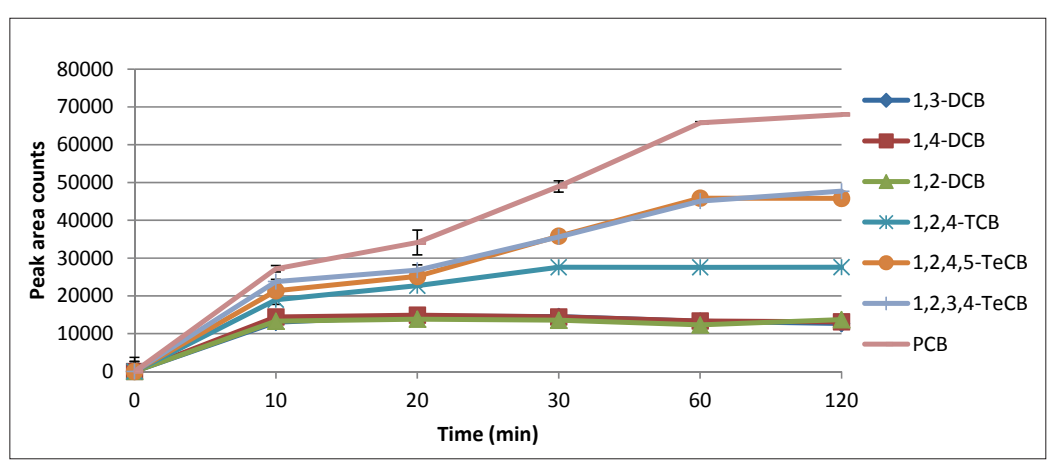

amount of water molecules available to dissolve the analytes, which results in an increased mass transfer of certain analytes into the extraction phase (He et al., 2000; Psillakis, 2002). He et al. (2000) reported an increase in extraction efficiency with ionic strength, in the extraction of chlorobenzenes from river water using HS-SPME. These authors reported an optimum ionic strength of $20 \%(\mathrm{~m} / \mathrm{v})$. In contrast, the optimum ionic strength obtained in this study was $5 \%(\mathrm{~m} / \mathrm{v})$. This discrepancy can be explained in terms of the mode of extraction used. He et al. (2000) used the headspace technique and thus the SPME fibre was not exposed to the ionic species and other interferences in the sample matrix. Since this study used the direct immersion mode the fibre was directly exposed to the ionic species and interferences in the sample matrix. Initially, an increase in extraction efficiency will be experienced (saltingout effect) at lower salt concentrations. However, further increases in salt concentration will result in a decrease in extraction efficiency. This is due to an increase in the interactions between the analytes and the ionic species, which reduces the analyte's ability to move into the extracting phase.

However, an addition of salt resulted in the introduction of several interferences in the sample matrix as can be seen in the chromatogram given as Fig. 6. Although there was no coelution, these interferences would have resulted in a decrease in both the limits of detection and quantification. Since the salting-out effect had a slight effect on the extraction efficiency, it was decided to carry out further experiments without the addition of any salt.

\section{Effect of duration of extraction}

SPME is an equilibrium process; therefore it is imperative to determine the time required for each analyte to attain equilibrium with the fibre coating. While maintaining all other extraction parameters at optimum levels, the duration of extraction was varied from $5 \mathrm{~min}$ to $120 \mathrm{~min}$. The results are depicted in Fig. 7.

The equilibration time is defined as the time after which the amount of analyte extracted remains constant (Pawliszyn, 1997). Figure 7 indicates that the DCB isomers attained equilibrium rapidly. 1,2,4-TCB required $30 \mathrm{~min}$ to attain equilibrium while 1,2,3,4-TeCB, 1,2,4,5-TeCB and $\mathrm{PCB}$ required $60 \mathrm{~min}$. The results indicate that equilibrium times are compound dependent, having a trend of increasing times with increasing degree of chlorination and molecular weight. The chlorobenzene compounds with higher vapour pressures and octanol-water partition coefficients and lower molecular mass attained equilibrium rapidly. To enhance throughput, a 30-min extraction was selected for the rest of this study. It should be noted that by terminating the extraction process prior to equilibration of PCB, 1,2,3,4- and 1,2,4,5-TeCB being attained, reduced method reproducibility can be anticipated. This occurs during 'pre-equilibrium' of analytes. Small changes in time result in large change in the amounts of analytes adsorbed (Vas and Vekey, 2004).

\section{Validation of the optimised DI-SPME method}

A newly-developed analytical method needs to be validated to determine its suitability for the intended application. The figures of merit of the validation experiments are shown in Table 1.

\section{Linearity}

The linearity of the analytical method was determined within an analyte mass range of $1-100 \mathrm{ng} / \mathrm{m} \ell$. The $R^{2}$-values of the analytes ranged from $0.9957-0.9995$ (Table 1). This indicates good linearity of the optimised method as there is a linear relationship 


\begin{tabular}{|c|c|c|c|c|c|c|c|c|}
\hline \multicolumn{9}{|c|}{$\begin{array}{c}\text { Table } 1 \\
\text { Figures of merit obtained during method validation }\end{array}$} \\
\hline Compound & $\begin{array}{l}\text { Linearitya } \\
\left(R^{2}-\text { value }\right)\end{array}$ & $\underset{\text { LOD }}{\text { LOD })}$ & $\begin{array}{c}\text { LOQ } \\
(\mathrm{ng} / \mathrm{m} \ell)\end{array}$ & $\begin{array}{l}\text { Recovery }^{b} \\
\text { (\%) }\end{array}$ & $\% \mathbf{R S D}^{b}$ & $\begin{array}{c}\text { WHO } \\
\begin{array}{c}\text { Guidelines } \\
\text { (ng/me) }\end{array}\end{array}$ & $\begin{array}{c}\text { USEPA Drinking } \\
\text { Water Guidelines }{ }^{d} \\
\text { (ng/me) }\end{array}$ & $\begin{array}{l}\text { LOD } \\
(n g / m \ell)\end{array}$ \\
\hline 1,3-DCB & 0.9987 & 0.0204 & 0.2048 & 105.6 & 2.2 & - & - & - \\
\hline 1,4-DCB & 0.9957 & 0.0429 & 0.4293 & 102.4 & 1.9 & 300 & 75 & 0.1 \\
\hline 1,2-DCB & 0.9995 & 0.0219 & 0.2193 & 101.4 & 5.6 & 1000 & 600 & 0.1 \\
\hline $1,2,4-\mathrm{TCB}$ & 0.9984 & 0.2650 & 2.650 & 93.4 & 0.6 & - & 70 & 0.5 \\
\hline $1,2,4,5-\mathrm{TeCB}$ & 0.9966 & 0.0910 & 0.9105 & 83.6 & 9.2 & - & - & - \\
\hline $1,2,3,4-\mathrm{TeCB}$ & 0.9975 & 0.0730 & 0.7305 & 102.8 & 0.5 & - & - & - \\
\hline PCB & 0.9996 & 0.0720 & 0.7203 & 107.2 & 7.2 & - & - & - \\
\hline
\end{tabular}

$a-$ Five-point linear range $(1,10,25,50,100 \mathrm{ng} / \mathrm{m} \ell)$

$b-n=3$; spiking level $5 \mathrm{ng} / \mathrm{m} \ell$

$c-W H O, 2011$

d-USEPA, 2003

$e$ - Limit of detection obtained from Zhao et al. (2009)

Figure 8

(a) Dam sample

fortified with $5 \mathrm{ng} / \mathrm{ml}$ of each analyte;

(1) 1,3-DCB; (2) 1,4-

$D C B$; (3) $1,2-D C B$;

(4) $1,2,4-T C B$,

(5) $1,2,4,5-T e C B$

(6) $1,2,3,4-T e C B$ (7) $P C B$;

(b) Dam sample blank
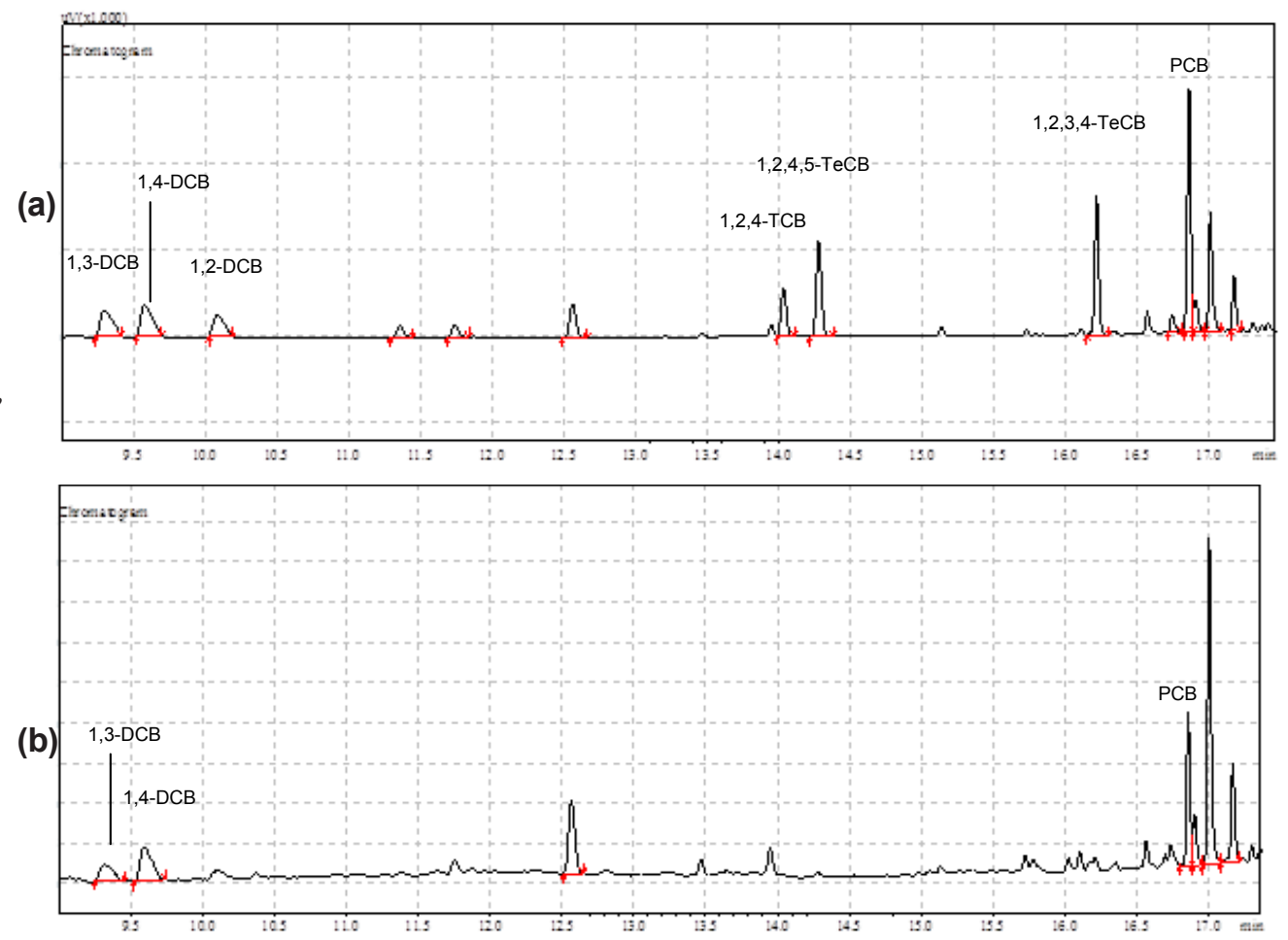

between analyte mass and detector response within the range of trace concentration levels. He et al. (2000) reported correlation coefficients of greater than 0.9910 for each analyte, at a linearity range of $0.2-20 \mathrm{ng} / \mathrm{m} \ell$ using HS-SPME coupled to GC-MS. This study produced slightly improved correlation coefficients, of greater than 0.9957 , over a wider concentration range, indicating that there is a more accurate mathematical relationship between detector response and analyte concentration.

\section{Limits of detection and quantification}

As generally accepted, the LOD was taken to be the concentration of analyte which yielded a signal to noise ratio of 3 , and the LOQ as the concentration of analyte which yielded a signal to noise ratio of 10. Table 1 shows the LOD and LOQ obtained for each analyte. Furthermore, the LOQs obtained in this study are well below the regulatory limits of the World Health Organisation (WHO) and USEPA drinking water guidelines. This indicates that the LOQs obtained in this study are well within the levels required for trace concentration analysis and are suitable for the routine analysis of chlorobenzenes in drinking water.

\section{Recovery experiments}

Recovery experiments were performed to determine the efficiency of the method for the extraction of chlorobenzene from the Grootdraai Dam water samples, as well as to investigate the presence of any matrix interferences. The optimised method was initially applied to $5 \mathrm{~m} \ell$ of water collected from the Grootdraai Dam. The chromatogram in Fig. $8 \mathrm{a}$ indicates the presence of 1,3-DCB, 1,4-DCB and PCB. The optimised method was then applied to $5 \mathrm{~m} \ell$ of Grootdraai Dam water which was fortified with $10 \mathrm{ng} / \mathrm{m} \ell$ of each analyte (Fig. 8b). The average recoveries obtained in this study were within acceptable limits ranging between $83.6 \%$ and $107.32 \%$ with RSD values of less than $10 \%$. This indicates that the optimised method is quantitative, free of any matrix interferences and has 


\begin{tabular}{|l|l|l|l|}
\hline \multicolumn{3}{|c|}{ Comparisons with other studies } \\
\hline Reference & $\begin{array}{l}\text { Sample preparation and detection } \\
\text { method }\end{array}$ & $\begin{array}{l}\text { Extraction } \\
\text { time } \\
\text { (min) }\end{array}$ & $\begin{array}{l}\text { Number of analytes, } \\
\text { Linear range (ng/me), } \\
\text { Range of recoveries, } \\
\text { \% Relative standard deviation } \\
\text { Limit of detection (ng/m })\end{array}$ \\
\hline Vidal et al. (2005) & $\begin{array}{l}\text { Single drop micro-extraction } \\
\text { (SDME); } \\
\text { GC-MS }\end{array}$ & 5 & $10,0.2-50,97 \%$ mean, 2.1-13.2\%, 0.003-0.031 \\
\hline Vidal et al. (2007) & $\begin{array}{l}\text { Micro-wave assisted headspace } \\
\text { (HS)-SDME; HPLC }\end{array}$ & $10-20$ & 8, not specified, 99\% mean, 2.3-8.3\%, 0.016-0.039 \\
\hline Li et al. (2010) & Novel SPME coating; GC-ECD & 15 & $\begin{array}{l}7, \text { not specified , 91\% mean, 2.1-4.9\%, 0.00032 } \\
-0.00225\end{array}$ \\
\hline $\begin{array}{l}\text { Bagheri and Aghakhani } \\
(2011)\end{array}$ & $\begin{array}{l}\text { Novel nanofibre coating - HS-SPME } \\
\text {; GC-MS }\end{array}$ & $\begin{array}{l}\text { Not } \\
\text { specified }\end{array}$ & not specified, 0.050-1, 94-102\%, 3-8\%, 0.010 \\
\hline Zhao et al. (2009) & SDME, FC-FID & 20 & $5,1-1000,88.9-110.9 \%, 7.73-12.39 \%, 0.1-0.5$ \\
\hline This study (2012) & Direct immersion SPME GC-FID & 30 & $7,1-100,83.6-107.2 \%, 0.5-9.2 \%, 0.020-0.265$ \\
\hline
\end{tabular}

\begin{tabular}{|l|c|c|}
\hline \multicolumn{3}{|c|}{$\begin{array}{c}\text { Table 3 } \\
\text { Water use trends for the Grootdraai Dam } \\
\text { (Adapted from Strauss, 2006) }\end{array}$} \\
\hline User & $\begin{array}{c}\text { Demand } \\
\mathbf{m}^{3} / \mathbf{a}\end{array}$ & $\begin{array}{c}\text { Return } \\
\mathbf{m}^{3} / \mathbf{a}\end{array}$ \\
\hline Irrigation & 321500 & - \\
\hline Power Station A & 47420000 & - \\
\hline Power Station B & 53838000 & - \\
\hline Industry A & 91250000 & 4015000 \\
\hline Municipality A & 3600000 & 1982124 \\
\hline Municipality B & 5420250 & 3011250 \\
\hline Thuthukane Township & 1427556 & 642400 \\
\hline
\end{tabular}

good repeatability. However, raw water samples, having interfering compounds such as humic and fulvic acids, may require additional clean-up procedures to improve the efficiency of the pre-concentration technique.

\section{Comparisons with literature reports}

The data from 5 reports published in journals have been tabulated in Table 3 to indicate the relative rank and value of the method reported herein compared with reputedly more sophisticated and sensitive methods. Although the LODs for the GC-FID method are much higher, relative to the studies listed Table 3 (Vidal et al., 2005; Vidal et al., 2007; Li et al., 2010; Bagheri and Aghakhani, 2011), it is worth noting that these LODs are adequate for monitoring samples to assess compliance with regulatory limits specified in international standards and guidelines (WHO in this case). In addition, the GC-FID is easy to use and is relatively inexpensive. However, upon comparison with a study using a similar detection system coupled to HS-SDME (Zhao et al., 2009), the LOD obtained in this study for 1,4-DCB, 1,2-DCB and 1,2,4-DCB is 1.9-4.6-fold lower. Additionally, the method presented in this study offers a higher degree of precision. This indicates that the DI-SPME method developed in this study is superior to the HS-SDME method of pre-concentration.

\section{Occurrence of chlorobenzenes in the Grootdraai Dam}

Three chlorobenzene isomers were detected in the Grootdraai Dam. These included 1,3-DCB; 1,4-DCB and PCB, at

concentrations of $0.429 \mathrm{ng} / \mathrm{m} \ell, 1.685 \mathrm{ng} / \mathrm{m} \ell$ and $1.433 \mathrm{ng} / \mathrm{m} \ell$, respectively. The major water users of the Grootdraai Dam are industries and municipalities (Table 2).

The contamination by chlorobenzene isomers in the Grootdraai Dam could be due to agricultural chemical run-off, indiscriminate discharge of effluent from chemical industries, air emissions during chemical manufacturing, return flow from wastewater treatment plants or disposal of materials containing these compounds within the catchment of this impoundment. 1,3-DCB and 1,4-DCB are commonly used in the manufacture of pesticides, indicating that agricultural activities within the catchment which use these pesticides are a possible source (McPherson et al., 2002). Various environmental influences, particularly stormwater run-off, can mobilise the chlorobenzenes which will ultimately enter the waterways emptying into the Grootdraai Dam through surface runoff.

The occurrence of PCB in the Grootdraai Dam is quite noteworthy, as there is currently no large-scale use and commercial production of the chemical. However, since PCB is produced in small quantities during the chlorination of benzenes, one can anticipate the presence of $\mathrm{PCB}$ when DCB is detected. Historically, PCB has been used in the manufacture of fungicides, and, more recently, as a flame retardant. This could indicate past PCB contamination, which has been remobilised through environmental processes, and eventually entered and accumulated in the Grootdraai Dam.

Domestic and industrial discharge could also be a source of chlorobenzenes. These compounds can be introduced into the Grootdraai Dam via return flows from wastewater treatment plants within the catchment. 1,4-DCB is used in common household products such as air fresheners, moth balls and urinal deodorisers. These chlorobenzene compounds are also common components of industrial degreasing solvents (McPherson et al., 2002). Negligent and poor waste disposal protocols at both domestic and industrial level can lead to contamination. The major industries in the Grootdraai catchment are are the Matia and Tutuka power stations and Sasol, Secunda. Table 2 indicates that both power stations have a zero-discharge policy; as a result, contamination is negligible. Sasol, as well as the Thutukane, Bethal and Ermelo Municipalities, are the main contributors to the return flow into the Grootdraai Sam. As a result, one cannot rule out these as possible sources of chlorobenzene contamination. 


\section{Comparison to drinking water guidelines}

The South African Drinking Water Standard (SANS) 241:2005 for drinking water does not regulate any of the detected chlorobenzenes. The SANS 241:2005 is derived from the World Health Organisation (WHO) Guidelines for drinking water quality. The SANS 241:2005 standard indicates that, for the organic determinants which are not listed, one should refer to the WHO Guidelines for drinking water for comparative purposes (DWAF, 2005). The WHO has set a health-based guideline value of $300 \mathrm{ng} / \mathrm{m} \ell$ for 1,4-DCB (WHO, 2011). This indicates that the level of 1,4-DCB within the Grootdraai Dam $(1.685 \mathrm{ng} / \mathrm{m} \ell)$ is well within the WHO guideline and does not pose a significant health risk. Since WHO has not derived a health-based guideline for the other two isomers detected in this study; a health risk assessment cannot be derived.

\section{Conclusions}

The results show that the technique of immersing the SPME fibre coupled with analysis by GC-FID is viable for routine monitoring of dam water samples for the presence of chlorinated benzenes. Furthermore, it is adequate to detect the limits set for two of the three chlorobenzenes regulated by the USEPA and for one of the two chlorobenzenes listed by the WHO. Furthermore, the optimised DI-SPME method produced lower LODs than the HS-SDME. An added advantage of this method is that it is relatively cheap and easy to use. It should therefore be of service to organisations which monitor their drinking water, as well as source water, for levels of chlorobenzenes, in order to ensure public health protection and safety of its customers.

\section{Acknowledgements}

Mr Jimmy Chetty is thanked for technical assistance and Eskom and DUT for financial support.

\section{References}

BAGHERI H and AGHAKHANI A (2011) Novel nanofibre coatings prepared by electrospinning technique for headspace solid-phase microextraction of chlorobenzenes from environmental samples. Anal. Methods 3 1284-1289.

CHISVERT A, ROMAN IP, VIDAL L and CANALS A (2009) Simple and commercially readily-available approach for the direct use of ionic liquid based single drop micro-extraction prior to gas chromatography: Determination of chlorobenzenes in real water samples as model analytical application. J. Chromatogr. A 1216 1290-1295.

DWAF (DEPARTMENT OF WATER AFFAIRS AND FORESTRY, SOUTH AFRICA) (2005) Drinking water quality management guide for water services authorities. Version: September 2005. Department of Water Affairs and Forestry, Pretoria.

FILHO AM, DOS SANTOS FN and PEREIRA PAP (2010) Development, validation and application of a method based on DI-SPME and GC-MS for the determination of pesticides of different chemical groups in surface and groundwater samples. Microchem. J. 96 139-145.

GROSJEAN D (1991) Atmospheric fate of toxic aromatic compounds. Sci. Total Environ. 100 367-414.

HE Y, WANG Y and LEE HK (2000) Short communication: Trace analysis of ten chlorinated benzenes in water by headspace solidphase microextraction. J. Chromatogr. A 874 149-154.

HUANG Y, YANG YC and SHU YY (2007) Analysis of semi-volatile organic compounds in aqueous samples by microwave-assisted headspace solid-phase microextraction coupled with gas-chromatography-electron capture detection. J. Chromatogr. A 1140 35-43.
JELENI A and MARE H (2007) Orange River integrated water resources management plan: Review of existing infrastructure in the Orange River Catchment. Report by WRP Consulting Engineers, Jeffares and Green, Sechaba Consulting, WCE (Pty.) Ltd. and Water Surveys Botswana (Pty.) Ltd., November 2007.

JUNIOR JLR and RE POPPI N (2007) Determination of organochlorine pesticides in groundwater samples using solid phase microextraction by gas chromatography-electron capture detection. Talanta 72 (5) 1833-1841.

KHAJEH M, YAMINI Y and HASSAN J (2006) Trace analysis of chlorobenzenes in water samples using headspace solvent microextraction and gas chromatography/electron capture detection. Talanta 69 (5) 1088-1094.

KIN CM (2008) Development and validation of a solid phase microextraction method for simultaneous determination of pesticide residues in fruits and vegetables by gas chromatography. $\mathrm{PhD}$ thesis, Faculty of Science, University of Malaya, Malaysia.

KOZANI RR, ASSADI Y, SHEMIRANI F, HOSSEINI MRM and JAMALI MR (2007) Part-per-trillion determination of chlorobenzenes in water using dispersive liquid-liquid microextraction combined gas chromatography-electron capture detection. Talanta 72 387-393.

LI Q, WANG X and YUAN D (2009) Preparation of solid phase microextraction fibre coated with single-walled carbon nanotubes by electrophoretic deposition and its application in extracting phenols from aqueous samples. J. Chromatogr. A 1216 (9) 1305-1311.

LI XJ, Y CW, HUO XL and ZENG Z (2010) Solid-phase microextraction using a diglycidyloxycalix[4]arene coated fibre combined with gas chromatography: very simple, rapid and sensitive method for the determination of chlorobenzenes in water. Microchimica Acta 168 161-167.

LIU G, WANG J, ZHU Y and ZHANG X (2004) Application of multiwalled carbon nanotubes as a solid phase sorbent for chlorobenzenes. Anal. Lett. 37 (14) 3085-3104.

LOPEZ-DARIAS J, PINO V, ANDERSON JL, GRAHAM CM and AFONSO AM (2010a) Determination of water pollutants by direct immersion solid phase microextraction using polymeric ionic liquid coatings. J. Chromatogr. A 1217 (8) 1236-1243.

LOPEZ-DARIAS J, PINO V, MENG Y, ANDERSON JL and AFONSO AM (2010b) Utilization of a benzyl functionalized polymeric ionic liquid for the sensitive determination of polycyclic aromatic hydrocarbons; parabens and alkyphenols in waters using solid phase microextraction coupled to gas chromatography-flame ionization detection. J. Chromatogr. A 1217 (46) 7189-7197.

LUAN T, FANG S, ZHONG Y, LIN L, CHAN SMN, LAN C and TAM NFY (2007) Determination of hydroxyl metabolites of polycyclic aromatic hydrocarbons by fully automated solid phase microextraction derivatization and gas chromatography-mass spectroscopy. J. Chromatogr. A 1173 (1-2) 37-43.

McPHERSON CA, TANG A, CHAPMAN PM, TAYLOR LA and GORMICAN SJ (2002) Toxicity of 1,4-dichlorobenzene in sediments to juvenile polychaete worms. Mar. Pollut. Bull. 44 (12) 1405-1414.

MENG Y and ANDERSON JL (2010) Tuning the selectivity of polymeric ionic liquid sorbent coatings for the extraction of polycyclic aromatic hydrocarbons using solid phase microextraction. $J$. Chromatogr. A 1217 (40) 6143-6152.

MONTERO L, POPP P, PASCHKE A and PAWLISZYN J (2004) Polydimethylsiloxane rod extraction, a novel technique for the determination of organic micropollutants in water samples by thermal desorption-capillary gas chromatography-mass spectrometry. J. Chromatogr. A 1025 17-26.

ORMAD P, CORTES S, SARASA J, MARTIN A, PUIG A and OVELLEIRO JL (1996) Characterization of organic micropollutants in the River Cinca by using granular activated carbon and liquid liquid extraction. J. Chromatogr. A 733 159-169.

PSILLAKIS E and KALOGERAKIS N (2002) Developments in single-drop microextraction. Trends Anal. Chem. 21 (1) 53-63.

PAWLISZYN J (1997) Solid phase microextraction (SPME). Chem. Educ. 2 (4) 1-7.

SANTOS FJ, GALCERAN MT and FRAISSE D (1996) Application of solid-phase microextraction to the analysis of volatile organic 
compounds in water. J. Chromatogr. A 742 181-189.

SOONTHORNTANTIKUL W, LEEPIPATPIBOON N, IKEGAMI T,

TANAKA N and NHUJAK T (2009). Selectivity comparisons of monolithic silica capillary columns modified with poly(octodecyl methacrylate) and octadecyl moieties for halogenated compounds in reversed-phase liquid chromatography. J. Chromatogr. A 1216 5868-5874.

STRAUSS KJ (2006) Application of pinch technology in water resource management to reduce water use and wastewater generation for an area. WRC Report No. 1241/1/06. Water Research Commission, Pretoria.

TOR A (2006) Determination of chlorobenzenes in water by dropbased liquid-phase microextraction and gas chromatography-electron capture detection: A short communication. J. Chromatogr. A 1125 129-132.

USEPA (UNITED STATES ENVIRONMENTAL PROTECTION AGENCY) (2003) National Primary Drinking Water Regulations. USEPA, Washington.

VAN GINKEL CE (2001) Toxic algal incident in the Grootdraai Dam. Internal Report no. N/C110/02/DEQ/0401. Institute for Water Quality Studies, Department of Water Affairs and Forestry, Pretoria.
VAS G and VEKEY K (2004) Solid-phase microextraction: a powerful sample preparation tool prior to mass spectrometric analysis. $J$. Mass Spectrom. 39 233-254.

VIDAL L, CANALS A, KALOGERAKIS N and PSILLAKIS E (2005) Headspace single-drop microextraction for the analysis of chlorobenzenes in water samples. J. Chromatogr. A 1089 25-30.

VIDAL L, PSILLAKIS E, DOMINI CE, GRANE N, MARKEN F and CANALS A (2007) An ionic liquid as a solvent for headspace single drop microextraction of chlorobenzenes from water samples. Anal. Chim. Acta 584 189-195.

WANG D, XING J, PENG J and WU C (2003) Novel benzo-15-crown-5 sol gel coating for solid phase microextraction. J. Chromatogr. A 1005 1-12.

WANG Y and LEE HK (1998) Determination of chlorobenzenes in water by solid-phase extraction and gas chromatography-mass spectrometry. J. Chromatogr. A 803 219-225.

WHO (WORLD HEALTH ORGANISATION) (2011) Guidelines for Drinking Water Quality ( $4^{\text {th }}$ edn.). World Health Organisation, Geneva.

ZHAO F, LU S, DU W and ZENG B (2009) Ionic liquid-based headspace single-drop microextraction coupled to gas chromatography for the determination of chlorobenzene derivatives. Microchim. Acta 165 29-33. 